UR-1621

$\mathrm{ER} / 40685 / 959$

January 2001

\title{
Progress Towards a Generator for BFKL Physics
}

\author{
Lynne H. Orr* and W.J Stirling ${ }^{\dagger}$ \\ * Department of Physics and Astronomy \\ University of Rochester \\ Rochester, NY 14627-0171 \\ ${ }^{\dagger}$ Institute for Particle Physics Phenomenology \\ University of Durham \\ Durham DH1 3LE, UK
}

\begin{abstract}
.
In certain regions of phase space in jet production, large logarithms can arise which are resummed by the BFKL equation. Linear colliders can potentially be excellent places to study BFKL effects in jet production. We discuss an approach to BFKL calculations which incorporates kinematic effects explicitly and can be implemented in an event generator.
\end{abstract}

\section{INTRODUCTION}

In certain kinematic regimes for some QCD processes, each power of the strong coupling constant $\alpha_{s}$ is accompanied by a large logarithm and fixed order perturbation theory fails. To calculate anything meaningful, the large logs must be resummed. The BFKL equation [1] resums these large logs - which are due to multiple real and virtual gluon emissions - for the regime where the gluons have comparable transverse momenta but are strongly ordered in rapidity.

This BFKL physics comes into play at high energy linear $e^{+} e^{-}$colliders in virtual photon scattering. The electron and positron emit virtual photons, which then scatter, producing multiple jets. The leading order QCD process is $\gamma^{*} \gamma^{*} \rightarrow q \bar{q} q \bar{q}$ via gluon exchange, and the corresponding BFKL process has a gluon ladder attached to the $t$-channel gluon. BFKL applies when the invariant mass $W$ of the hadronic system is large, and

$$
s>>Q^{2}>>\Lambda_{Q C D}^{2},
$$


where $s$ is the square of the center of mass energy and $Q^{2}$ is the virtuality of the photons. Physically, this corresponds to $e^{+} e^{-} \rightarrow e^{+} e^{-}+$hadrons, where the final electron and positron are scattered at low angles (forward scattering).

\section{IMPROVED BFKL APPROACH}

The BFKL equation can be solved analytically in very special circumstances. The analytic solutions involve summing over arbitrary numbers of gluons and integrating over arbitrarily large gluon transverse energies. Only leading order kinematics are included and there is no kinematic cost to emit gluons. It is difficult to reach appropriately asymptotic regions in experiments, so it is no surprise that BFKL predictions often overshoot data.

A solution to this problem has been obtained by solving the BFKL equation iteratively, making the gluon sum explicit so that kinematic cuts can be applied directly and the solution can be implemented in a Monte Carlo program [2]; see also [3]. The BFKL equation contains separate integrals over real and virtual emitted gluons. We can reorganize the equation by combining the 'unresolved' real emissions - those with transverse momenta below some minimum value (in practice chosen to be small compared to the momentum threshold for measured jets) - with the virtual emissions. We perform the integration over virtual and unresolved real emissions analytically. The integral containing the resolvable real emissions is left explicit. The resulting subprocess cross section is

$$
d \hat{\sigma}=d \hat{\sigma}_{0} \times \sum_{n \geq 0} f_{n}
$$

where $f_{n}$ is the iterated solution for $n$ real gluons emitted and contains an overall form factor due to virtual and unresolved emissions.

We have done this in [2], and incorporated the running of $\alpha_{s}$ as well. We applied the Monte Carlo to dijet production at large rapidity separation at the Tevatron, improving the agreement between the BFKL prediction and experiment for the azimuthal decorrelation between the jets.

\section{VIRTUAL $\gamma \gamma$ SCATTERING AT $E^{+} E^{-}$COLLIDERS}

Returning to virtual photon scattering at linear colliders, we also consider the leading order QCD processes from which the BFKL must be built. It is convenient to consider the differential cross section

$$
W^{2} Q_{1}^{2} Q_{2}^{2} \frac{d^{3} \sigma}{d W^{2} d Q_{1}^{2} d Q_{2}^{2}}
$$

as a function of $W^{2} / Q^{2}$. As shown in Figure 1, the four-quark cross section approaches a constant as $W^{2} / Q^{2}$ gets large, but the analytic BFKL solution (open 


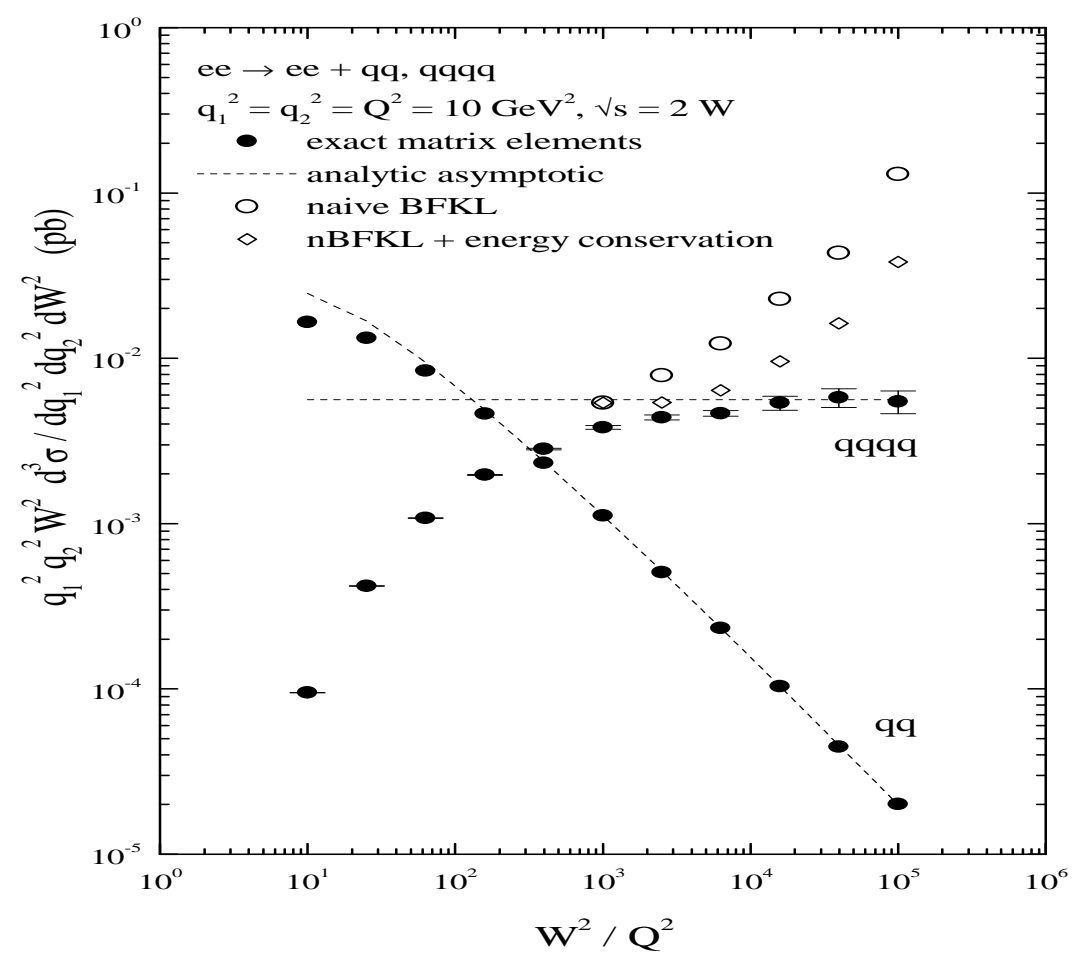

FIGURE 1. Exact (closed data points) and analytic asymptotic (dashed line) $e^{+} e^{-} \rightarrow e^{+} e^{-} q \bar{q}$ and $e^{+} e^{-} \rightarrow e^{+} e^{-} q \bar{q} q \bar{q}$ cross sections versus $W^{2} / Q^{2}$ at fixed $W^{2} / s=1 / 4$. Also shown: analytic BFKL without (open circles) and with (open diamonds) energy conservation imposed.

circles) rises. Also shown (open diamonds) is BFKL with energy conservation imposed on the emitted gluons, but without the entire machinery of the improved BFKL Monte Carlo; the result shown can be considered an upper limit on the improved BFKL result. An important point to note here is that the origin (in $W^{2} / Q^{2}$ ) of the BFKL curve, viz., the point where it begins to rise from the asymptotic QCD result, is arbitrary at leading order. We have made what seems a reasonable choice (where the QCD result is approaching its asymptotic value), but the entire BFKL curve can in principle be shifted horizontally.

Experimentally we are more interested in the cross section for fixed $\sqrt{s}$; this is shown as a function of $W$ in Figure 2 for $\sqrt{s}=500 \mathrm{GeV}$. In this case all of the cross sections fall off at large $W$, but the BFKL cross section lies above the others. The BFKL Monte Carlo calculation is in progress [4].

\section{COMMENTS ON LEP RESULTS}

Finally, we note that this process has been investigated at LEP. The L3 collaboration have measured the $\gamma^{*} \gamma^{*}$ cross section from double-tagged $e^{+} e^{-}$events, and their result lies between asymptotic QCD (which is flat) and analytic BFKL (which rises); see for example [5]. It is likely that the BFKL Monte Carlo prediction will lie closer to the data, but we note that the flat QCD prediction is the asymptotic 


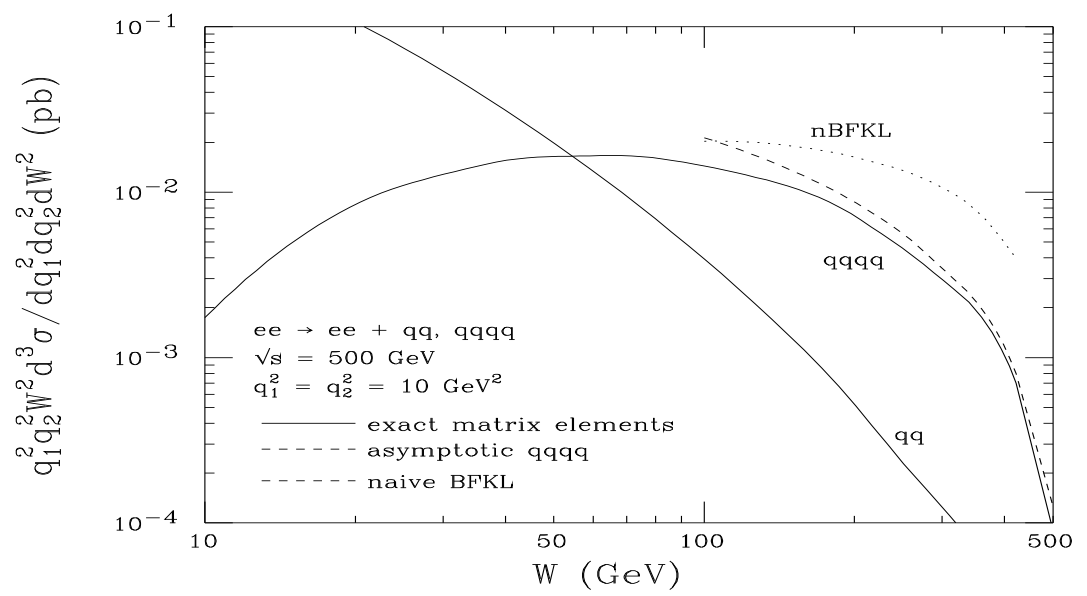

FIGURE 2. Exact (solid lines) and analytic asymptotic (dashed line) $e^{+} e^{-} \rightarrow e^{+} e^{-} q \bar{q}$ and $e^{+} e^{-} \rightarrow e^{+} e^{-} q \bar{q} q \bar{q}$ cross sections versus $W^{2} / Q^{2}$ at fixed $\sqrt{s}=500 \mathrm{GeV}$. Also shown: analytic BFKL (dotted line).

result. In fact the QCD prediction is rising in this region and has not reached the asymptotic limit. Until the fixed-order QCD and BFKL Monte Carlo predictions are sorted out, it is not clear exactly what we can conclude from the data. This work, at both LEP and linear collider energies, is currently in progress [4].

\section{REFERENCES}

1. L.N. Lipatov, Sov. J. Nucl. Phys. 23 (1976) 338; E.A. Kuraev, L.N. Lipatov and V.S. Fadin, Sov. Phys. JETP 45 (1977) 199; Ya.Ya. Balitsky and L.N. Lipatov, Sov. J. Nucl. Phys. 28 (1978) 822.

2. L.H. Orr and W.J. Stirling, Phys. Rev. D56 (1997) 5875.

3. C.R. Schmidt, Phys. Rev. Lett. 78 (1997) 4531.

4. L.H. Orr and W.J. Stirling, in progress.

5. See, for example, A. Csilling, proceedings of 2000 Meeting of the Division of Particles and Fields of the APS, Columbus, OH, August 9-12, 2000 (hep-ex/0011070) and references therein. 